\title{
Selection of Implants in Unilateral Prosthetic Breast Reconstruction and Contralateral Augmentation
}

\author{
Soo Jung Kim, Seung Yong Song, Dae Hyun Lew, Dong Won Lee \\ Institute for Human Tissue Restoration, Department of Plastic and Reconstructive Surgery, Yonsei University College of Medicine, Seoul, \\ Korea
}

Background In breast reconstruction using implants after unilateral mastectomy, it is challenging to create a natural, ptotic contour, and asymmetry is a potential drawback. To achieve breast symmetry and an ideal shape for both breasts, we performed contralateral augmentation in patients undergoing breast reconstruction with implants.

Methods Patients underwent unilateral mastectomy and 2-stage reconstruction. During the second stage of the procedure, contralateral augmentation mammoplasty was performed. Preoperatively, we obtained the patients' demographic information, and we then assessed breast volume, the volume and dimensions of the inserted implants, and complications. Breast symmetry was observed by the surgeon and was assessed by measuring the disparity between the final volume of each breast.

Results Contralateral augmentation was performed in 52 cases. When compared to patients who did not undergo a contralateral balancing procedure, patients who received contralateral augmentation were younger, thinner, and had smaller breasts. During implant selection for contralateral augmentation, we chose implants that were approximately $1 \mathrm{~cm}$ shorter in width, 1 level lower in height, and 1 or 2 levels lower in projection than the implants used for reconstruction. The postoperative breast contours were symmetric and the final volume discrepancy between each breast, which was measured by 3-dimensional scanning, was acceptable.

Conclusions We demonstrate that contralateral augmentation can be recommended for patients who perceive their breasts to be small and not beautiful in order to achieve an ideal and beautiful shape for both breasts. Furthermore, this study offers guidelines for selecting the implant that will lead to the optimal aesthetic outcome.

Keywords Breast reconstruction / Mammaplasty / Breast implant
Correspondence: Dong Won Lee Institute for Human Tissue Restoration, Department of Plastic and Reconstructive Surgery, Yonsei University College of Medicine, 50-1 Yonsei-ro, Seodaemun-gu, Seoul 03722 , Korea

Tel: +82-2-2228-2215

Fax: +82-2-393-6947

E-mail:xyphoss@yuhs.ac

Received: 12 May 2017 • Revised: 6 Aug 2017 • Accepted: 19 Aug 2017

pISSN: 2234-6163・ elSSN: 2234-6171 • https://doi.org/10.5999/aps.2017.44.5.413・ Arch Plast Surg 2017;44:413-419

No potential conflict of interest relevant to this article was reported.

\section{INTRODUCTION}

As breast reconstruction after total mastectomy is commonly performed worldwide, reconstruction techniques using autologous tissue and implants are currently evolving. Previously, the goal of breast reconstruction was to design a breast mound that

Copyright (C) 2017 The Korean Society of Plastic and Reconstructive Surgeons

This is an Open Access article distributed under the terms of the Creative Commons Attribution Non-Commercial License (http://creativecommons.org/

licenses/by-nc/4.0/) which permits unrestricted non-commercial use, distribution, and reproduction in any medium, provided the original work is properly cited.

www.e-aps.org 
would allow a patient's clothes to fit; however, patients' expectations are now higher, and they want the volume and shape of their breasts to be symmetrical, just as their original breasts were before mastectomy. For this reason, the frequency of contralateral balancing procedures is increasing. Occasionally, moderately symmetric breasts can be achieved by only reconstructing the ipsilateral breast, without performing any procedure on the contralateral side. However, in some cases, such as when the contralateral breast is too large, too small, or too ptotic, it does not have a beautiful and ideal shape, making it difficult to achieve a symmetric volume and contour simply by reconstructing the ipsilateral breast after mastectomy. There are several options for the contralateral balancing procedure, including reduction mammoplasty, augmentation mammoplasty, and mastopexy. Using one of these procedures, a plastic surgeon can transform both breasts into their ideal shape.

Breast reconstruction can be divided into implant-based reconstruction and autologous tissue reconstruction. Each reconstruction method has its advantages and disadvantages, so determining the most suitable method for a patient should be done carefully, taking into consideration the patient's age, breast contour, body mass index (BMI), underlying disease, and her own desires. Regardless of the method chosen for breast reconstruction, it is not easy to achieve completely beautiful bilateral breasts, and in an effort to achieve symmetry, we are often compelled to operate on the contralateral breast as well. The purpose of a contralateral balancing procedure is to achieve an aesthetically beautiful and natural-appearing breast that is in balance with the opposite side, and through this, the patient can achieve personal satisfaction, improving her self-esteem and quality of life.

Many studies have been conducted of the timing of contralateral balancing procedures; in particular, comparisons have been made between performing a contralateral balancing procedure simultaneously, at the time of initial reconstruction, and performing it as a delayed, second-stage procedure [1-4]. At the authors' hospital, simultaneous prosthesis-based reconstruction after unilateral mastectomy is most commonly performed. This option is usually preferred by women who want a simple operation and short recovery time, and by those whose chief concern is donor-site scarring. These patients inevitably undergo a secondary operation, during which the previously inserted tissue expander is exchanged for a permanent implant. Therefore, if they have an imperfect contralateral breast and want their breasts to have a more symmetrical appearance, we perform a contralateral balancing procedure at the time of the second operation. Since Korean women often tend to have relatively small breasts, many patients express their desire for augmentation mammo- plasty along with a contralateral balancing procedure at the initial preoperative consultation. In this study, we analyzed the patients who underwent contralateral augmentation after breast reconstruction with implants, and evaluated which type of implants allowed the creation of the optimal aesthetic outcomes.

\section{METHODS}

\section{Patients and evaluation}

The medical records of patients who received implant-based reconstruction by a single surgeon at a single institution were reviewed retrospectively. A total of 188 patients underwent unilateral mastectomy and 2-stage reconstruction using tissue expanders and implants. Patients who received bilateral mastectomy, contralateral prophylactic mastectomy, or a direct-to-implant procedure simultaneously were excluded. The contralateral balancing procedures included augmentation mammoplasty, reduction mammoplasty, and mastopexy. Among these patients, we collected the preoperative demographic information of patients who received contralateral augmentation, and assessed breast volume and complications after the procedure. We also analyzed the volume and dimensions of the inserted implants, including width, height, and projection. We evaluated the aesthetic outcomes at outpatient follow-up visits, based on the findings of the surgeon's physical examination. In addition, clinical photos were taken and the degree of symmetry was estimated by measuring the discrepancy between the final volume of each side, as assessed by 3-dimensional scanning (Axis Three, AX3 Technologies, Miami, FL, USA).

\section{Surgical procedure}

The first stage of the operation was performed simultaneously with the total unilateral mastectomy done by the general surgery team, and we then inserted a tissue expander in the reconstructed breast and slung it with acellular dermal matrix. After sufficient expansion of the skin was achieved by the tissue expander, considering the patient's desired end volume, the second stage of the operation was performed. Patients who required adjuvant chemotherapy underwent a second operation when the chemotherapy was completed. The existing tissue expander was removed from the ipsilateral breast and a permanent implant was inserted in the dual plane pocket, consisting of submuscular and preexisting acellular dermal matrix. In the contralateral breast, we created a dual plane - of type I, II, or III-according to the degree of ptosis. The augmentation approach was either transaxillary or via the inframammary fold. After inserting salinefilled sizers in both breasts, patients were then placed in a sitting position to confirm the symmetry of size and shape. Subse- 
quently, anatomical silicone gel-textured implants were inserted in both breasts.

\section{RESULTS}

Among the 188 women who received 2-stage, implant-based reconstructions, 93 patients did not undergo a contralateral balancing procedure. Ninety-five patients $(50.5 \%)$ received a contralateral balancing procedure to create a more beautiful contralateral breast and to improve breast symmetry. Among the patients who received a contralateral balancing procedure, 52 (54.7\%) underwent augmentation mammoplasty, 8 (8.4\%) underwent reduction mammoplasty, and 35 (36.8\%) underwent mastopexy (Fig. 1). Augmentation mammoplasty was the most common contralateral procedure. The mean age of the patients who received augmentation mammoplasty was 40 years, ranging from 30 to 59 years. The mean BMI was $20.1 \mathrm{~kg} / \mathrm{m}^{2}$, ranging from 17.21 to $25.86 \mathrm{~kg} / \mathrm{m}^{2}$. Most patients were slender and thin, as the BMI of 45 patients (86.5\%) was under $23 \mathrm{~kg} / \mathrm{m}^{2}$. The mean volume of the preoperatively measured breasts was $218 \mathrm{~mL}$ on the side of the lesion, and $221 \mathrm{~mL}$ on the healthy side. In contrast, the mean age of the patients who received no contralateral procedure was 44 years, which was older than that of patients who underwent contralateral augmentation. The mean BMI of these patients was $22.4 \mathrm{~kg} / \mathrm{m}^{2}$, which was higher than that of patients who underwent contralateral augmentation. The mean volume of the preoperatively measured breasts was also greater, with volumes of $315 \mathrm{~mL}$ on the side of the lesion and $317 \mathrm{~mL}$ on the healthy side (Table 1). Compared to patients who received no contralateral procedure, patients who received contralateral augmentation mammoplasty were younger, thinner, and had smaller breasts. As their original breasts before mastectomy were not ideally shaped due to insufficient breast volume, they were able to achieve beautiful and properlysized breasts by simultaneously undergoing contralateral aug-

\section{Fig. 1. Proportion of contralateral balancing procedures} performed

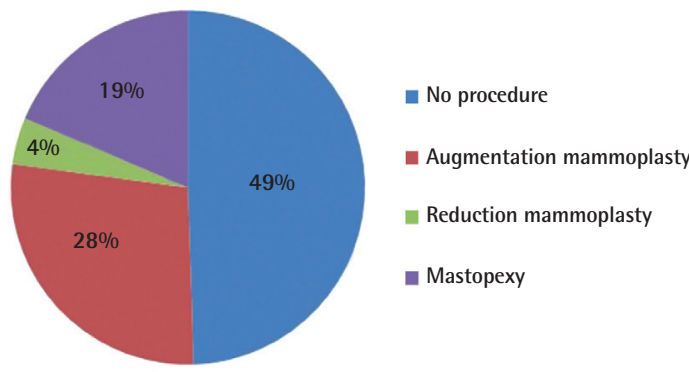

mentation.

We mainly used anatomical silicone gel-textured implants. Before the second stage of the operation, we had to consider the implant dimensions, including width, height, projection, and volume, to choose the implant that would achieve the optimal results. The average size of the implants inserted into the postmastectomy breast being prepared for reconstruction was 375 $\mathrm{mL}$, ranging from 245 to $495 \mathrm{~mL}$. The average size of the implants that were inserted into the contralateral breast for augmentation was $190 \mathrm{~mL}$, ranging from 90 to $280 \mathrm{~mL}$ (Table 2). The operating surgeon chose an implant dimension for the contralateral breast that would be 1 level lower than the implant placed on the post-mastectomy side, taking into consideration each breast's width, height, and projection. We selected the implant width for the post-mastectomy breast according to the patient's chest wall width and the width of the preexisting tissue expander. Subsequently, we chose an implant for the contralateral breast, the width of which was approximately $1 \mathrm{~cm}$ shorter than that of the reconstructed breast. The average difference in width between each implant in a single patient was $1.35 \mathrm{~cm}$. In terms of implant height and projection, most manufactured implants are divided into 3 levels of height — short, medium, and tall - and 4 levels of projection - low, medium, high, and extrahigh. The surgeon endeavored to select implants for contralateral augmentation that were 1 level lower in height and 1 or 2 levels lower in projection than the implants used for breast reconstruction, a decision that took skin thickness into consideration. The implants that were used for reconstruction were mostly tall

\section{Table 1. Patient demographics}

\begin{tabular}{|lcc|}
\hline & $\begin{array}{c}\text { Contralateral } \\
\text { augmentation } \\
(\mathbf{n}=\mathbf{5 2})\end{array}$ & $\begin{array}{c}\text { No procedure } \\
(\mathrm{n}=\mathbf{9 3})\end{array}$ \\
\hline Age $(\mathrm{yr})$ & 40 & 44 \\
Body mass index $\left(\mathrm{kg} / \mathrm{m}^{2}\right)$ & 20.1 & 22.4 \\
Preoperative 3D volume $(\mathrm{mL})$ & & \\
$\quad$ Ipsilateral breast & 218 & 315 \\
$\quad$ Contralateral breast & 221 & 317 \\
\hline
\end{tabular}

Table 2. Volume of the implants used

\begin{tabular}{|cc|}
\hline Implant volume & No. (\%) \\
\hline Implant volume in reconstructed breast & \\
$200-295 \mathrm{~mL}$ & $5(9.6)$ \\
300-395 mL & $24(46.1)$ \\
400-495 mL & $23(44.2)$ \\
Implant volume in contralateral breast & \\
$<100 \mathrm{~mL}$ & $2(3.8)$ \\
$100-195 \mathrm{~mL}$ & $28(53.8)$ \\
$200-295 \mathrm{~mL}$ & $22(42.3)$
\end{tabular}


in height, and high or extra-high in their projection. The implants that were used for contralateral augmentation were mostly medium-height and medium-projection implants. Tables 3 and 4 show the dimensions of the implants that we used for each breast. On average, the height of the implant used for augmentation was 1.02 levels lower than that of the implant used for reconstruction, and the projection of the implant used for augmentation was 1.45 levels lower than that of the implant used for reconstruction.

The minimum follow-up period was 3 months, and the mean follow-up period was 11.7 months. Three months after the final operation, clinical photographs and a 3-dimensional scan were taken at the outpatient clinic. The average final volume discrepancy, which was $15.7 \mathrm{~mL}$, was determined by calculating the difference between the 3-dimensionally measured volume of

\begin{tabular}{|c|c|c|c|c|}
\hline & Low proj & Mid proj & High proj & Ex-high proj \\
\hline Short height & 0 & 0 & 0 & 0 \\
\hline Mid height & 0 & $1(0.02)$ & $5(9.6)$ & 0 \\
\hline Tall height & 0 & $4(7.7)$ & $22(42.3)$ & $20(38.5)$ \\
\hline
\end{tabular}

Values are presented as number (\%). each breast. This is not a noticeable difference to the naked eye, and thus, the results were deemed acceptable (Table 5). The overall satisfaction was high in the evaluation of the operating surgeon according to the patients' subjective observations (Figs. $2,3)$. There were no serious complications from infection, hematoma, or implant malposition; there were only 2 cases involving minor complications among the 52 patients. One was a

Table 4. Dimensions of implants inserted into the contralateral augmented breast

\begin{tabular}{|lcccc|}
\hline & Low proj & Mid proj & High proj & Ex-high proj \\
\hline Short height & $1(0.02)$ & $2(4.6)$ & 0 & 0 \\
Mid height & $5(9.6)$ & $36(69.2)$ & $5(9.6)$ & 0 \\
Tall height & 0 & $3(5.8)$ & 0 & 0 \\
\hline \multicolumn{4}{l}{ Values are presented as number (\%). } \\
\hline
\end{tabular}

Table 5. Three-dimensional volume discrepancy between the breasts

\begin{tabular}{|lr|}
\hline 3-Dimensional volume discrepancy $(\mathrm{mL})$ & No. $(\%)$ \\
\hline$<10$ & $28(53.8)$ \\
$10-50$ & $23(44.2)$ \\
$>50$ & $1(1.92)$ \\
\hline
\end{tabular}

Fig. 2. A case of a 35-year-old woman

A $450-\mathrm{mL}$ implant, the width of which was $13.0 \mathrm{~cm}$, with tall height and extra-high projection, was inserted into the right breast for reconstruction. A $280-\mathrm{mL}$ implant, the width of which was $12.0 \mathrm{~cm}$ with medium height and medium projection, was inserted into the left breast for augmentation. (A-C) Preoperative photographs. The breast volumes measured by 3-dimensional scanning were $150 \mathrm{~mL}$ and $156 \mathrm{~mL}$. (D-F) One-year postoperative photographs. The breast volumes measured by 3 -dimensional scanning were $462 \mathrm{~mL}$ and $464 \mathrm{~mL}$.
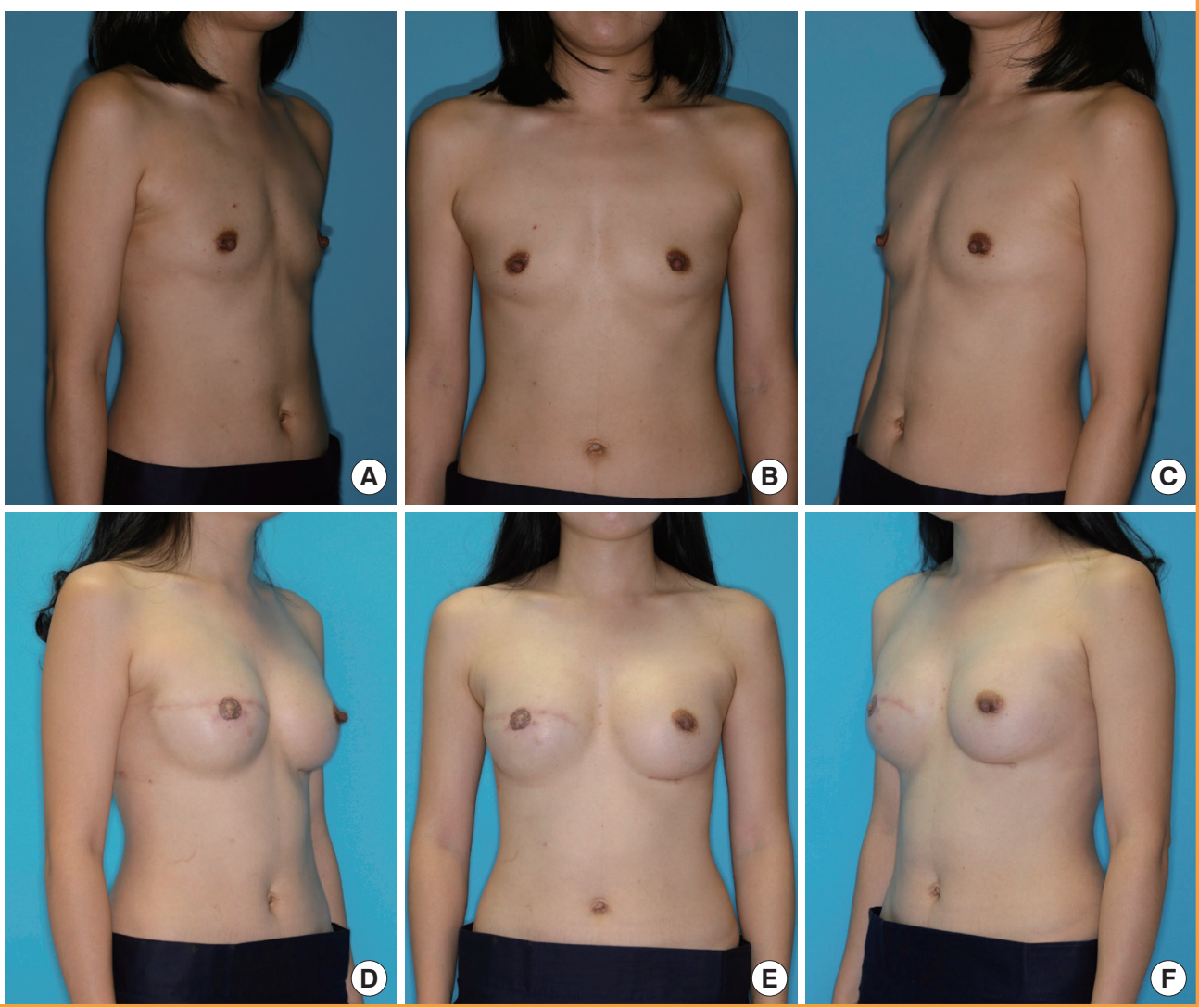


\section{Fig. 3. A case of a 36-year-old woman}

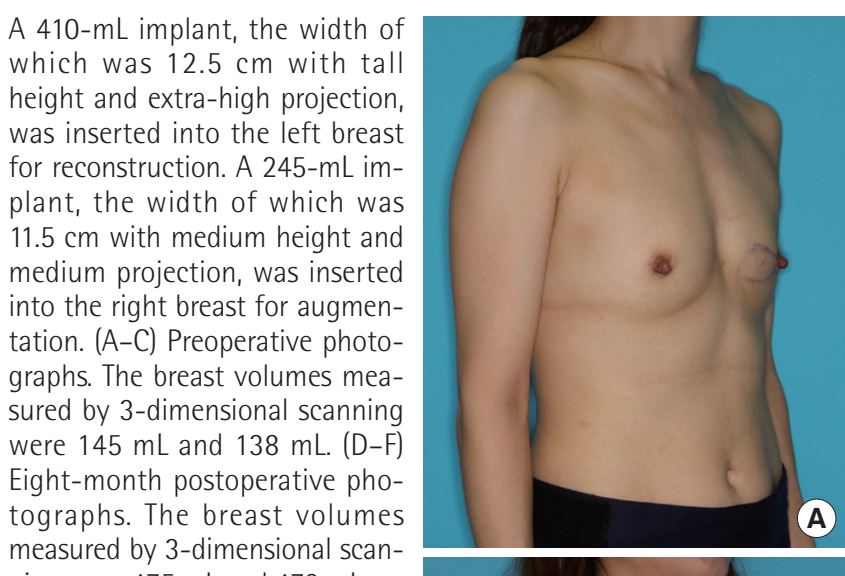

measured by 3-dimensional scanning were $475 \mathrm{~mL}$ and $472 \mathrm{~mL}$.

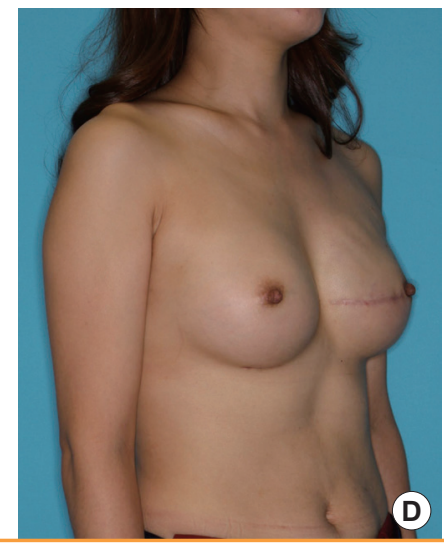

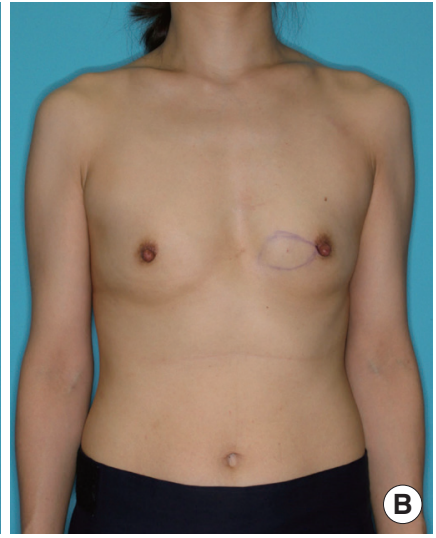
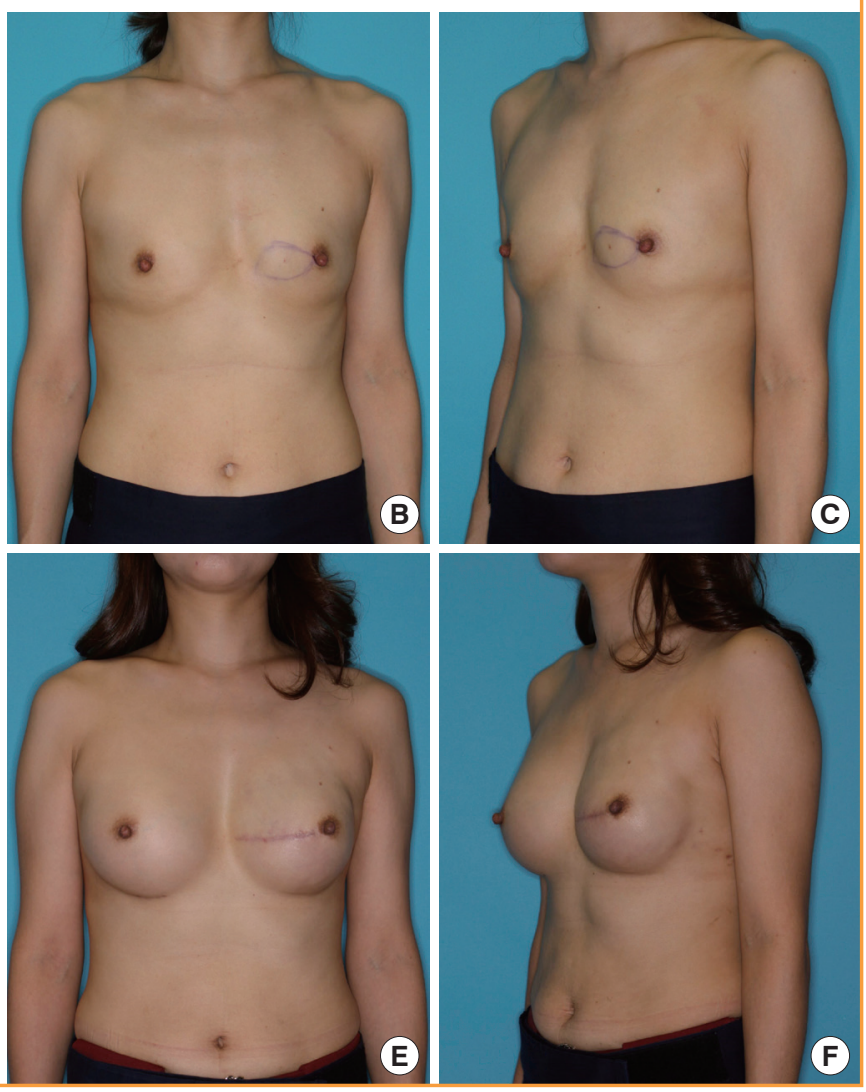

postoperative infection, which healed well with antibiotic treatment and did not necessitate the removal of the implant, and the other was a malpositioned implant, which was straightforwardly corrected by reposition surgery. Complications requiring reoperation were not reported in any other cases.

\section{DISCUSSION}

Contralateral balancing procedures are commonly performed to achieve symmetry with a reconstructed breast; however, whether it is preferable to perform them during immediate reconstruction or during the delayed second stage is still controversial [1-4]. Some reasons for performing a contralateral balancing procedure during immediate reconstruction are that the second-stage operation can be avoided, and that the time period that patients live with asymmetric breasts (which may cause them to experience depression until the second procedure is performed) can be reduced. However, if a direct-to-implant procedure and contralateral augmentation are performed simultaneously, the remaining skin flap after mastectomy may be at risk when an excessively large implant is inserted at once. Instead, if a contralateral balancing procedure is performed as a delayed operation, patients can determine the preferred size of the im- plant carefully during the interval between the first-stage operation and the second-stage operation.

In Giacalone's [5] study, 50\% of patients who received delayed reconstruction needed a contralateral secondary procedure to obtain breast symmetry. Losken et al. [3] reviewed 1,394 patients who underwent reconstruction, and found that $67 \%$ needed a contralateral symmetry procedure after delayed reconstruction, while $22 \%$ needed it after immediate reconstruction. The incidence of a contralateral procedure was higher in cases of implant-based reconstruction than in cases of autologous tissue reconstruction. The most common procedure after implantbased reconstruction was contralateral augmentation, whereas after autologous tissue reconstruction, reduction mammoplasty was the most popular procedure. Implant-based reconstruction required more balancing procedures than autologous tissue reconstruction because it is more difficult to achieve contour symmetry without a secondary procedure in implant-based reconstruction due to its unnaturalness. Nahabedian [2] retrospectively reviewed 382 patients who received breast reconstruction. In that study, balancing procedures were more commonly performed in autologous tissue reconstructions, and they argued that autologous tissue was more amenable than an implant when a secondary procedure was performed. 
In Nahabedian and Losken's study, they performed contralateral balancing procedures as delayed operations; however, in the study of Smith et al. [4], they performed them simultaneously with breast reconstruction. Smith et al. tried to create breast symmetry during the first operation. One of the main advantages of autologous tissue reconstruction is that a second operation can be avoided, but if a second procedure is required, then that advantage disappears. They argued that a single-stage approach could reduce the number of operations, cost of admission and operation, period required for recuperation, and the period that a patient's breasts remain asymmetric, which can be prolonged when a patient needs adjuvant therapy. However, a disadvantage of implant-based reconstruction is capsular contracture, which can deform the breast shape and lead to asymmetry. Therefore, if a contralateral balancing procedure is performed at the same time as immediate implant-based reconstruction, a new asymmetry will appear later, and then a secondary procedure may be unavoidable.

Surgeons who argue that it is better to offer a contralateral balancing procedure as a delayed, secondary procedure think that if the shape of a breast becomes different from the immediate postoperative result under the influence of postoperative chemotherapy or ongoing fat necrosis, they can consider other surgical options during the second-stage operation. These may include not only the performance of a contralateral procedure, but also an additional procedure in the ipsilateral breast, such as necrotic fat excision, microfat grafting, or inframammary fold repositioning, to achieve a more perfectly balanced breast shape.

In this study, we used 3-dimensional imaging to estimate breast volume and to assess breast symmetry. We took digital photographs and 3-dimensional scans routinely in every patient who underwent breast reconstruction preoperatively, after the first operation, and after the second operation. Many studies have already reviewed 3-dimensional imaging, and it is not $100 \%$ accurate; however, its advantage is that we can determine the approximate volume of each breast and estimate the difference between the volume of the breasts, thus determining the amount of reduction or augmentation required in the contralateral breast [6-9].

As other mastectomy options developed, in contrast to the traditional radical mastectomy, the methods and timing (delayed to immediate) of reconstruction also progressed. In the past, delayed reconstruction was widespread, but presently, immediate reconstruction is the more preferred method because it has several advantages. It provides good aesthetic results and psychological benefits, it does not postpone adjuvant therapy, and it does not have a negative effect on patient outcomes. It does not increase local cancer recurrence or affect breast cancer survival
$[10,11]$. However, a reconstructive surgeon should not overlook the remaining oncologic risk on the contralateral side when performing an operation [12]. When there is cancer in one breast, the potential risk that cancer will be found in the contralateral breast in the future is not great, but it still exists, and we cannot ignore it. The likelihood that breast cancer will appear in the contralateral side is $6 \%$ after 10 years and $9 \%$ after 20 years [ 13 16]. Therefore, a surgeon should explain this possibility to patients and evaluate the opposite breast for cancer development properly by regular physical examinations, mammography, and magnetic resonance imaging before surgery [17]. Furthermore, a surgeon should recommend regular oncologic surveillance during the postoperative period. It has been suggested that implant-based augmentation has the disadvantage of reducing the sensitivity of mammography, but this usually happens when the implant is inserted in a subglandular plane $[18,19]$. This can be overcome when the implant is placed under the pectoralis major muscle; in this case, the breast tissue can be stretched by the volume of the implant, and it becomes easier to palpate for the detection of a possible mass. According to previous studies, augmentation mammoplasty as a contralateral balancing procedure does not delay the detection or diagnosis of newly developed cancer and it does not affect the prognosis of cancer [1]. If patients are made aware of the oncologic issue and agree to receive regular cancer screening, a contralateral balancing procedure is an effective operation for breast symmetry that can be recommended.

Patients' expectations and the desire to achieve breast symmetry after breast reconstruction are increasing. In this study, augmentation mammoplasty was most commonly used as a contralateral balancing procedure because Korean women are more slender and have more hypoplastic breasts than Western women. Performing contralateral augmentation mammoplasty can provide patients with heightened self-esteem and satisfaction, and improve their quality of life through the achievement of breast symmetry. Patients who receive a 2 -stage procedure will inevitably need a second operation, so it is recommended to perform the contralateral procedure at the second stage in order to achieve an ideal and beautiful shape in both breasts. Furthermore, this study offers guidelines for the selection of implant dimensions (width, height, and projection) that will achieve the optimal aesthetic outcomes.

\section{PATIENT CONSENT}

The patient provided written informed consent for the publication and the use of their images. 


\section{REFERENCES}

1. Nahabedian MY. Managing the opposite breast: contralateral symmetry procedures. Cancer J 2008;14:258-63.

2. Nahabedian MY. Symmetrical breast reconstruction: analysis of secondary procedures after reconstruction with implants and autologous tissue. Plast Reconstr Surg 2005;115: 257-60.

3. Losken A, Carlson GW, Bostwick J 3rd, et al. Trends in unilateral breast reconstruction and management of the contralateral breast: the Emory experience. Plast Reconstr Surg 2002;110:89-97.

4. Smith ML, Clarke-Pearson EM, Vornovitsky M, et al. The efficacy of simultaneous breast reconstruction and contralateral balancing procedures in reducing the need for second stage operations. Arch Plast Surg 2014;41:535-41.

5. Giacalone PL, Bricout N, Dantas MJ, et al. Achieving symmetry in unilateral breast reconstruction: 17 years experience with 683 patients. Aesthetic Plast Surg 2002;26:299302.

6. Nahabedian MY, Galdino G. Symmetrical breast reconstruction: is there a role for three-dimensional digital photography? Plast Reconstr Surg 2003;112:1582-90.

7. Tepper OM, Small K, Rudolph L, et al. Virtual 3-dimensional modeling as a valuable adjunct to aesthetic and reconstructive breast surgery. Am J Surg 2006;192:548-51.

8. Losken A, Fishman I, Denson DD, et al. An objective evaluation of breast symmetry and shape differences using 3-dimensional images. Ann Plast Surg 2005;55:571-5.

9. Lee WY, Kim MJ, Lew DH, et al. Three-dimensional surface imaging is an effective tool for measuring breast volume: a validation study. Arch Plast Surg 2016;43:430-7.
10. Singletary SE, Kroll SS. Skin-sparing mastectomy with immediate breast reconstruction. Adv Surg 1996;30:39-52.

11. Kroll SS, Khoo A, Singletary SE, et al. Local recurrence risk after skin-sparing and conventional mastectomy: a 6-year follow-up. Plast Reconstr Surg 1999;104:421-5.

12. Eccles DM, Gareth Evans D. Management of the contralateral breast in patients with hereditary breast cancer. Breast 2000;9:301-5.

13. Raabe NK, Sauer T, Erichsen A, et al. Breast cancer in the contralateral breast: incidence and histopathology after unilateral radical treatment of the first breast cancer. Oncol Rep 1999;6:1001-7.

14. Healey EA, Cook EF, Orav EJ, et al. Contralateral breast cancer: clinical characteristics and impact on prognosis. J Clin Oncol 1993;11:1545-52.

15. Claus EB, Stowe M, Carter D, et al. The risk of a contralateral breast cancer among women diagnosed with ductal and lobular breast carcinoma in situ: data from the Connecticut Tumor Registry. Breast 2003;12:451-6.

16. Rosen PP, Groshen S, Kinne DW, et al. Contralateral breast carcinoma: an assessment of risk and prognosis in stage I (T1N0M0) and stage II (T1N1M0) patients with 20-year follow-up. Surgery 1989;106:904-10.

17. Mendelson EB. Evaluation of the postoperative breast. Radiol Clin North Am 1992;30:107-38.

18. Silverstein MJ, Handel N, Gamagami P, et al. Mammographic measurements before and after augmentation mammaplasty. Plast Reconstr Surg 1990;86:1126-30.

19. Handel N, Silverstein MJ, Gamagami P, et al. Factors affecting mammographic visualization of the breast after augmentation mammaplasty.JAMA 1992;268:1913-7. 